(Invited - 9th Gonference on the Applications of Accelerators in Research and Industry, Nov. 10-12, 1986, Denton, TX)

\title{
SURGERY OF FAST, HIGHLY CHARGED IONS STUDIED B̄ï ZERO-DEGREE AUGER SPECTROSCOPY
}

N. STOLTERFOHT* ${ }^{*}$, P.D. MILLER, H.F. KRAUSE, Y. YAMAZAKI\#, J.K. SWENSON, R. BRUCH ${ }^{+}$, P.F. DITTNER, P.L. PEPMILLER, and S. DATZ

Oak Ridge National Laboratory, Physics Division

P. O. Box, Oak Ridge, TN 37830

CONF-861114--36

DE87 003655

Lero-degree Auger spectra were measured in collisions of oxygen and carbon on He with incident charge states of $q=2$ to 5 and for energies from 5 to $30 \mathrm{MeV}$. Since the light target particle He acts selectively on the projectile ion, we refer to the present method as ion surgery. Apart from the one-electron processes single excitation and single loss, two-electron processes such as transfer excitation and transfer loss are studied.

\section{Introduction}

The study of highly charged ions has received considerable attention in the past few years. An important method to obtain detailed information about multiply charged ions is the spectroscopy of Auger electrons produced in energetic ion-atom collisions. In particular, the use of the projectile Auger spectroscopy [1] at high-energy accelerators has the advantage that high incident charge states may selectively be produced by stripping and magnetically analyzing the projectile prior to the experiment. Generaliy, projectile Auger spectroscopy involves serious problems with kinematic line broadening effects which can, however, essentially be avoided by measuring the Auger electrons under che observation angle of $\%$. Recent experiments $[2,3]$ indicate the method of zero-degree Auger spectroscopy [4] yields excellent spectral resolution at incident energies as high as $100 \mathrm{MeV}$.

Another serious problem involved in Auger spectroscopy is the copious number of states produced in violent ion-atom collisions. The number of sta- 
tes can be significantly reduced by using light target atoms such as hydrogen or helium [2]. Low $Z$ particles have the ability to act as a 'needle' in selectively ionizing the inner shell without substantially disturbing the outer shells. Thus, for instance, the outer-shell configuration of the projectile ${ }_{\wedge}^{\text {is }}$ preserved during needle ionization so that the number of charge states produced is limited. This needle ionization, exploited by Itoh et al. $[2,3]$, may be considered as a specific example of the more general concept of ion surgery. [5]

The method of ion surgery involves ionization, excitation, and capture of one or, a most, two electrons in a fast, highly stripped projectile without affecting its other electrons. The idea is to consider the projectile ion not as a simple point source for a Coulomb force but as a structured object whose properties can be investigated by the action of a light incident target particle. Ion surgery in conjunction with zero-degree Auger spectroscopy allows for the conclusive identification of the projectile charge state after the collision obviatirig charge selective coincidence measurements [6]. In addition to that, high resolution Auger spectroscopy separates between specific energy levels excited in the collision. This state selectivity is the outstinding feature of the ion surgery used together with, the high resolution Auger spectroscopy.

The analysis of the Auger spectra yields information about the atomic structure [7] as well as information about the excitation mechanisms [8] of the inner-shell vacancy states involved. The atomic structure information may be used as a testing ground for fundamental theories including relativistic many-body problems [9]. In this case, the zero-degree Auge spectroscopy is particularly attractive since it yields results of unprecedented accuracy. Recently, Itoh et al. [2,3] and Bruch et al. [11] performed detailed atomic structure studies by comparing experimental and theoretical level energies. In contrast to this, the amount of work devoted to the excitation mechanisms using zero-degree Auger spectroscopy is still limited.

The present article emphasizes aspects of the reaction mechanism involved in collisions of multiply charged oxygen and carbon projectiles on He. The projectile energies used range from several MeV to several tens of MeV. At the high energy limit one-electron processes such as single excitation and 
single loss dominate. However, at decreasing projectile energy two-electron processes such as transfer excitation or transfer loss gain importance. Here, the ion surgery becomes an interesting and challenging task.

\section{The system $\mathrm{O}^{4+}+\mathrm{He}, \mathrm{O}^{3+}+\mathrm{He}$, and $\mathrm{O}^{2+}+\mathrm{He}$}

The experiments were performed at the Oak Ridge National Laboratory EN Tandem facility using the zero-degree Auger spectroscopy apparaturs temporarily transported from the Hahn-Meitner-Institut, Berlin. The apparatus has been described in detail before [2,3]. Fig. 1 displays $0-K$ Auger spectra obtained in collisions of $10-\mathrm{MeV} 0^{4+}, 0^{3+}$, and $0^{2+}$ with He. It is noted that the electron energy scale refers to the projectile rest frame and that the continuous electron background is subtracted. The experimental line energies are determined with the relatively high accuracy of $0.2 \mathrm{eV}$ which is obtained usig kinematic line doubling and compression effects $[4,5]$. The line assignment is discussed in detail elsewhere $[8,10]$.

In Table I results from the Auger spectroscopy spectra concerning various reaction mechanisms are shown. The total cross sections given in this work are obtained with the assumption that the emission of the Auger electrons is isotropic in the emitter rest frame. The experimental results may be compared with calculations using the binary encounter theory (BEA) [11] which has heen found to be accurate within $20 \%$ for the reversed collision systems [12]. For $0^{4+}$ impactthe BEA results compare reasonably well with the experimental data whereas for the other ious $0^{3+}$ and $0^{2+}$ theory and experiments shows discrepancies which are not understood at present. In the calculations $K-$ shell binding energies for ionized oxygen are used. It is noted that the use of such binding energies instead of those for the neutral atom effects the cross section by nearly a factor of 2 .

In Fig. 1 the lines labeled $\mathrm{A}, \mathrm{B}$, and $\mathrm{C}$ refer to $\mathrm{Li}-, \mathrm{Be}-$, and $\mathrm{B}-$ like states, respectively. The spectra show that the prominent lines are produced by needle ionization as pointed out previously for neon impact [3]. Thus, the most probable charge state of the emitting ion is one unit higher than the incident one (see also Table I). Similarly, needle excitation produces the projectile charge state equal to that of the incoming ion. Moreover, lines are also observed corresponding to a charge state which is two units higher 


\section{$-4-$}

than the incident one. The ocurrence of the double electron loss process indicates the limit for the applicability of the needie ionization concept. For $10-\mathrm{MeV} 0^{3+}$ and $0^{2+}$ the contributions from double electron loss are $21 *$ and $35 \%$, respectively (Table 1 ). This shows that the two-electron process are significant for the present collision systems. Nevertheless, the number of lines in the Auger spectra is still sufficiently small so that individual lines con separately be studied.

The state selectivity of the ion surgery method yields detailed information. As noted above, needle ionization favors the preservation of the outershell configuration during the collision. In Fig. 1 several states are seen where the outer shell is not in its ground state configuration. Hence, it may be inferred that a significant fraction of the incident beam is in a metastable state $[3,5]$. For example, the four-electron ion $0^{4+}$ is highly metastable in the state $1 \mathrm{~s}^{2} \mathrm{ss} 2 \mathrm{p}{ }^{3} \mathrm{P}$. It may be converted by $1 \mathrm{~s}$ ionization into the states $1 s 2 s 2 p{ }^{4} p$ (1 ine $\left.A 2\right)$ and $1 s\left(2 s 2 p{ }^{3} p\right)^{2} p$ (1 ine $\left.A 3\right)$ which clearly demonstrate how the incident (triplet) state of the outer shell is preserved during the collision. Similarly, the incident ground state $1 s^{2} 2 s^{2}{ }^{1} S$ is converted to $1 s 2 s^{2}{ }^{2} S$ (line $A 1$ ). From the intensity of the lines $A 2$ and $A 3$ relative to the intensity of all Li-like lines, one obtains a value of $65 \%$ for the fraction of metastable ions in the incident beam [13]. Similar considerations yield a fraction of $55 \%$ for the metastabel quartet states of the incident five-electron ions $0^{3+}$. These numbers which may be appear to be surprisingly large are understood when a statistical population of the multiplets is assumed [5].

Since two-electron processes are expected to occur in the present collision systems, it may be anticipated that in the system $0^{4+}+$ He the configuration $1 \mathrm{~s} 2 \mathrm{~s} 2 \mathrm{p}$ is produced by a loss-excitation process, i.e. by the loss of a is electron accompanied by a $2 s-2 p$ transition. To verify the importance of this two-electron process, one can use the fact that spin flip is negligible in the present collision systems. Hence, the loss-excitation process produces essentially the $1 s\left(2 s 2 p I_{P}\right)^{2} p$ state corresponding to the 1 ine A4 whose intensity, nowever, is seen to be small (see also Table I). Furthermore, the

state $1 s\left(2 s 2 p{ }^{3} p\right){ }^{2} p$ could be produced by $2 s$ electron loss accompanied by $1 s-2 p$ excitation. However, it is noted that this loss-excitation process cannot produce the $1 s 2 s 2 p{ }^{4} p$ state. 
The influence of the loss-excitation process may further be verified at higher energies where needle ionization works more efficiently. As expected it is found that the $1 \mathrm{~s}(2 \mathrm{~s} 2 \mathrm{p} \quad \mathrm{l} P)^{2} \mathrm{p}$ line is reduced when $25-\mathrm{MeV} \mathrm{O}^{4+}$ is ised instead of 10 -iMeV $0^{4+}$. On the other hand, the $1 \mathrm{~s}\left(2 \mathrm{~s} 2 \mathrm{p}{ }^{3} \mathrm{p}\right)^{2} \mathrm{p}$ line does not change in relative intensity as the projectile energy increases. This shows that the $1 s\left(2 s 2 p{ }^{3} p\right)^{2} p$ line is not much effected by the loss-excitation process. Rather, as pointed out before this state is expected to be due to the metastable fraction in the ion bean.

In a quantitative analysis of the line intensities it should be taken into account that the states designated to the Auger lines are only the main components among other ones. For example, the line A4 attributed to $1 s\left(2 s 2 p{ }^{1} p\right)^{2 p}$ contains a significant admixture of the state $1 s\left(2 s 2 p{ }^{3} p\right)^{2} p$. Similarly, the line $A 2$ designated to $1 s 2 s 2 p{ }^{4} p$ contains the ${ }^{2} p$ states as admixtures produced by the spin-orbit interaction. Further work is needed to study the mixing coefficients for the siates under considerations here.

\section{The system $0^{5+}+\mathrm{He}$}

The specific feature of $0^{5+}$ is that the single loss cannot be observed by means of Auger spectroscopy, as the states produced are not autoionizing. In this case the most prominent lines in the Augar spectra are produced by single excitation. This can be seen in Fig. 2 which displays $0^{5+}+$ He spectra for incident energies between $5 \mathrm{MeV}$ and $20 \mathrm{MeV}$. The contributions of different reaction mechanisms for this systems are given in Table I. Single excitation is responsible for the production of the Li-like states except for $1 s 2 s 2 p{ }^{4} p$. This quartet state is produced by a transfer-loss process where the 1 s electron is accompanied by the capture of a $2 p$ electron. Furthermore, the Be-like states are created by transfer excitation [14].

Fig. 3 shows cross sections for the production of various states produced in the $0^{5+}+$ He system. From Fig. $3 a$ it is seen that the dominant excitation process corresponds to the dipole transition $1 \mathrm{~s}-2 \mathrm{p}$. The related cross section curve exhibits a maximum whose position is understood from the well-known Massey criterion. A similar maximum shifted to lower energies is seen for the cross section curve refering to the quadrupole transition 1s-2s. 
Fig. 3b shows that the cross section for the production of the $1 \mathrm{~s} 2 \mathrm{~s} 2 \mathrm{p}{ }^{4} p$ is strongly enhanced at low energies. This behaviour is typical for electron transfer processes. It supports our interpretation that the quartet state is produced by a transfer-loss process. Similarly, the fact that the cross section for the production of the $1 s(2 s 2 p 1 p)^{2 p}$ state is enhanced at low energies may indicate that the transfer-loss process plays ${ }_{1}$ certain role in this case.

However, the enhanced production of the $1 s(2 s 2 p 1 p)^{2 p}$ state nay also be due to another second-order process. At low energies the $1 \mathrm{~s}-2 \mathrm{p}$ transition competes with the successive transitions $2 s-2 s$ and $2 s-2 p$. The one-step process and the two-step process are described by the first and second Born approximation, respectively. It is important to note that, contrary to the $1 s\left(2 s 2 p{ }^{1} p\right)^{2} p$ state, the $1 s\left(2 s 2 p{ }^{3} p\right)^{2} p$ state cannot be produced by the two-step process $1 s-2 s$ and $2 s-2 p$. (Because of the Pauli principle the electrons in the $2 \mathrm{~s}$ subshell cannot have parallel spins.) Hence, the cross section ratio of the ${ }^{2} p$ states may provide direct information about the contribution from the second Born term. It is noted that, in principle, this contribution may also be destructive.

Fig. $3 c$ shows the cross section data for the Be-like states $1 \mathrm{~s} 2 \mathrm{~s} 2 \mathrm{p}^{2}$ 10 $_{0}$ and $1 s 2 s 2 p^{2}{ }^{3} \mathrm{D}$ which are produced by transfer excitation. The interesting point with this process is that it may proceed in a resonant type manner, i.e. if the target electron is regarded as being free, transfer exciation is identical to a reversed Auger process which takes place via the electron-electron interaction. The resonant tranfer-excitation (RTE) process, first studied by Tanis et al. [15], as received particular attention in the past few years $[16,17]$. In Fig. 3c the data clearly show the resonant behaviour of the transfer-excitation process as a maximum in the cross section curve. The correspondence of RTE with the Auger process manifests itself by the fact that the maximum cross section occurs for a projectile velocity equal to the velocity of the Auger electron involved. Further information about the present tranffer-excitation data will be discussed in a forthcoming publication by Swenson et al. [14]. 
4. The system $\mathrm{C}^{2+}+\mathrm{He}, \mathrm{C}^{4+}+\mathrm{He}$, and $\mathrm{C}^{5+}+\mathrm{He}$

The Auger spectra obtained in collisions of multiply ionized carbon with He are displayed in Fig. 4. It is seen that the spectrum of the $\mathrm{C}^{2+}+$ He system is similar to that of the isoelectronic system $0^{4+}+\mathrm{He}$ (Fig. 1). The strong excitation of the states $1 s 2 s 2 p{ }^{4} p$ and $1 s\left(2 s 2 p{ }^{3} p\right)^{2} p$ indicates a significant fraction of metastable ions in the incident beam. The fraction of $75 \%$ obtained from the spectrum is even higher than that for oxygen.

The $\mathrm{C}^{4+}+$ He spectrum shows lines only due to Li-like states which are very likely produced by one-electron transfer to projectiles in the metastable state $152 \mathrm{~s}{ }^{3} \mathrm{~S}$. This has been pointed out previously by Dillingham et a1. [8]. In addition, the most prominent line $1 s 2 p^{2}{ }^{2} D$ is created by a transfer excitation process, i.e. the transfer into the $2 p$ shell is accompanied by a $2 s-2 p$ transition.

The spectrum for the incident one-electron ion $\mathrm{c}^{5+}$ exhibits only weak 1 ines for Li-like configurations which might be produced by double electron capture into shells with $n>2[8]$. Rather, at higher electron energies the spectrum shows one prominent line which is designated to the He-like states $2 p^{2} 1_{D}$ and/or $2 s 2 p{ }^{1} p$. These states are produced by transfer excitation as discussed by Itoh et al. [17] for $\mathrm{He}^{+}$impact. It is noted that the projectile energy of $7 \mathrm{MeV}$ is close tonthe cross section maximum for resonant transfer excitation. Future work is suggested to study the RTE process for incidert one-electron ions by means of high-resolution Auger spectroscopy.

\section{Acknowledgement}

We are indebted to Dieter Schneider and John Tanis for valuable discussions. This work was supported by the U.S. Deparmtent of Energy, Division of Basic Energy Sience under Contract No DE-AC05-840R21400 with Mart in Mariettà Energy Systems, Inc. 
$\underline{\text { References }}$

+ Permanent address: Hahn-Meitner-Institut Berlin, Glienickerstr. 100, D-1000 Berl in 39, Germany

\# Permanent address: Research Laboratory for Nuclear Reactors, Tokyo Institut of Technology, Tokyo, Japan

Permanent address: University of Nevada, Department of Physics, Reno NV 89557, USA

[1] I.A. Sellin, Topics in Current Physcis, Vol. I. Beam Foil Spectroscopy, Ed. S. Bashkin (Springer-Verlag, Heidelberg 1976) p. 265

[2] A. Itoh, T. Schneider, G. Schiwietz, H. Platten, G. Nolte, D. Schneider, and N. Stolterfoht, J. Phys. B 16 (1983) 3965

[3] A. Itoh, D. Schneider, T. Schneider, T.J.M. Zouros, G. Nolte, G. Schiwietz, W. Zeit.z, and N. Stolterfoht, Phys. Rev. 31 (1985) 684

[4] N. Stolterfoht, A. Itoh, D. Schneider, Th. Schneider, G. Schiwietz, H. Platten, G. Nolte, R. Glodde, U. Stettner, W. Zeitz, and T.J.M. Zouros, International Conference on $X$-Ray and Inner-Shell Processes in Atoms, Molecules and Solids, Invited Lecture, ed. A. Meisel (Leipzig 1984) p. 1983

[5] N. Stolterfoht, Phys. Reports 1986, in print

[6] S. Hagmann, C.L. Cocke, P. Richard, A. Skutlartz, S. Kelbch, H. Schmidt-Böcking, and R. Schuch, Electronic and Atom Collisions, eds. J. Eichler, I.V. Hertel, and N. Stolterfoht (North Holland, Amsterdam, 1984) p. 385 and references therein.

[7] R. Bruch, D. Schneider, W.H.E. Schwarz, M. Meinhardt, B.M. Johnson, and K. Taulbjerg, Phys. Rev. A19, 587 (1979) and references therein. 
[8] T.R. Dillingham, J. Newcomb. J. Hall, P.L. Pepmiller, and P. Richard, Phys. Rev. A 29 (1984) 3029 and references therein.

[9] C. A. Nicoequibles and T.Mercolcris, Phys. Rev. A 32 (1985) 3247 and references there in.

[10]R. Bruch: N. Stolterfoht, S. Datz, P.D. Miller, P.L. Pepiniller, J.K. Swenson,

Y. Yamazaki, and H.F. Krause, Phys. Rev. A (1986) to be published.

[11]J. D. Garcia, Phys. Rev. A 1 (1970) 280

[12]N. Stolterfoht and D. Schneider, Phys. Rev. A 11 (1975) 721

[13] The metastable fraction given by Itoh et al. [3] for $\mathrm{Ne}^{6+}$ impact is in error. The correct value of $51 \%$ is evaluated in Ref. 5.

[14]J.K. Srenson, Y. Yamazaki, P.D. Miiler, H.F. Krause, P.F. Dittner, P.L. Pepmiller, S. Datz, and N. Striterfoht, Phys. Rev. Lett. (1986) in print.

[15]A. Tanis, E.M. Bernstein, W.G. Graham, M.P. Stöckli, M. Clark, R.H. McFarland, T.J. Morgar, K.H. Berkner, A.S. Schlachter, J.W. Stearns, Phys. Rev. Lett. 53, (1984) 2551

[16]D. Brandt, Phys. Rev. A $\underline{27}$ (1983) 1314

[17]A. Itoh, T.J. Zouros, D. Schneide, U. Stettner, W. Zeitz, and N. Stolterfoht, J. Phys. B. 18 (1985) 4581

\section{DISCLAIMER}

This report was prepared as an account of work sponsored by an agency of the United States Government. Neither the United States Government nor any agency thercof, nor any of their employees, makes any warranty, express or implied, or assumes any legal liability or responsibility for the accuracy, completeress, or usefulness of any information, apparatus, product, or process disclosed, or represents that its use would not iníringe privately owned rights. Reference herein to any specific commercial product, process, or service by trade name, trademark, mariufacturer, or otherwise does not necessarily constitute or imply its endorsement, recommendation, or favoring by the United States Government or any agency thereof. The views and opinions of authors expressed herein do not necessarily state or reflect those of the United States Government or any agency thereof. 
Table I: Absolute cross section for the production of $0-k$-Auger electrons in 10-MeV $\mathrm{Oq}^{+}+$He collisions and reAvrive contribution of various rêction mechanisms. The error the absolute values is $\pm 30 \%$

\begin{tabular}{|l|cccc|}
\multicolumn{1}{|c}{ Mechanisms } & $0^{5+}$ & $0^{4+}$ & $0^{3+}$ & $0^{2+}$ \\
\hline Single Excitation & $69 \%$ & $27 \%$ & $9 \%$ & small \\
Single Loss & - & $67 \%$ & $67 \%$ & $62 \%$ \\
Transfer Excitation & $17 \%$ & small & smal & - \\
Transfer Loss & $11 \%$ & - & - & - \\
Loss Excitation & - & $4 \%$ & - & - \\
Double Excitation & $3 \%$ & - & - & - \\
Double Loss & - & - & $21 \%$ & $35 \%$ \\
\hline Total Cross Section & $\vdots$ & & & \\
{$\left[10^{\left.-19 \mathrm{~cm}^{2}\right]}\right.$} & 2.9 & 4.4 & 3.5 & 2.4 \\
\hline
\end{tabular}


Figure Captions

Fig. 1 Auger spectra in $10 . \mathrm{MeV} 0^{+}+$He col 1 isions with $q=2,3$, and 4

Fig. 2 Auger spectra produced in $0^{5+}+$ He collisions for different incident energies. The Be-like states 1 abeled $D$ and $\bar{D}$ refer to the decay to the final configuration $1 s^{2} 2 s$ and $1 s^{2} 2 p$, respectively.

Fig. 3 Cross sections for the production of Auger electrons for different initial states produced in 5 -to $30-\mathrm{MeV}^{5+}+$ He collisions.

Fig. 4 Auger spectra produced in 5 -and $7-\mathrm{MeV} \mathrm{C}^{\mathrm{q}^{+}}+$He collisions with $q=2,4$, and 5 . The labels are as in Fig. 1 . 


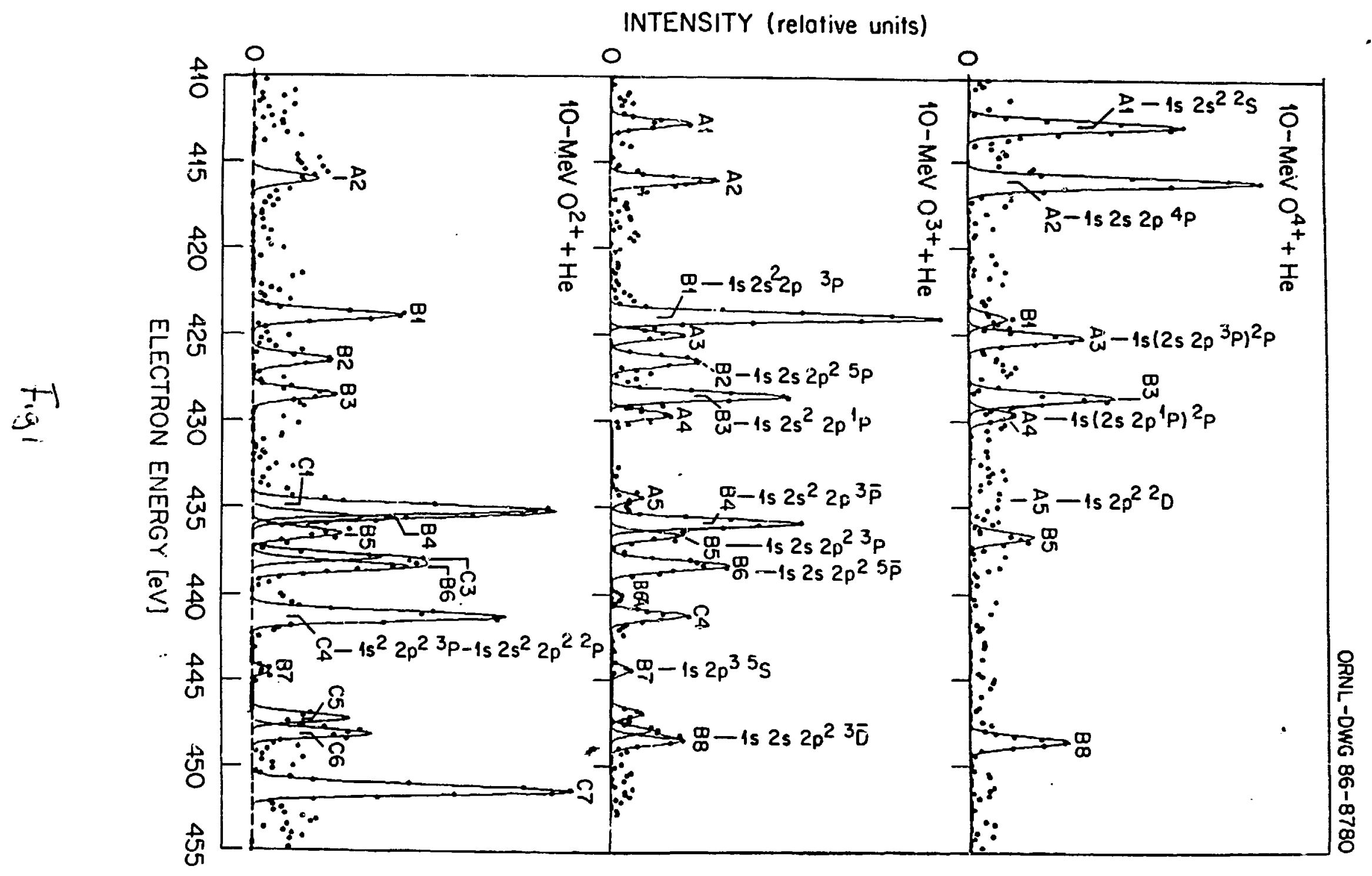




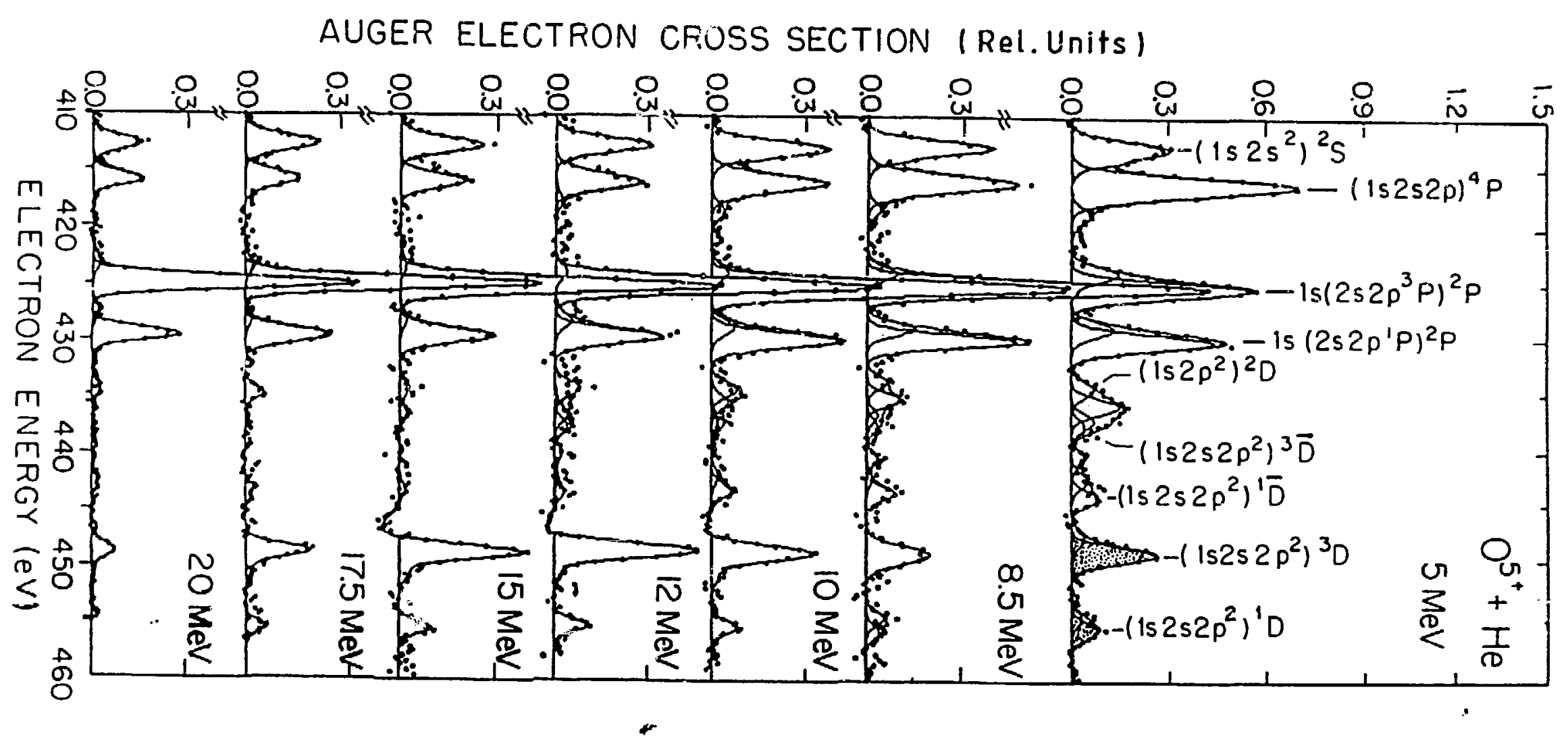




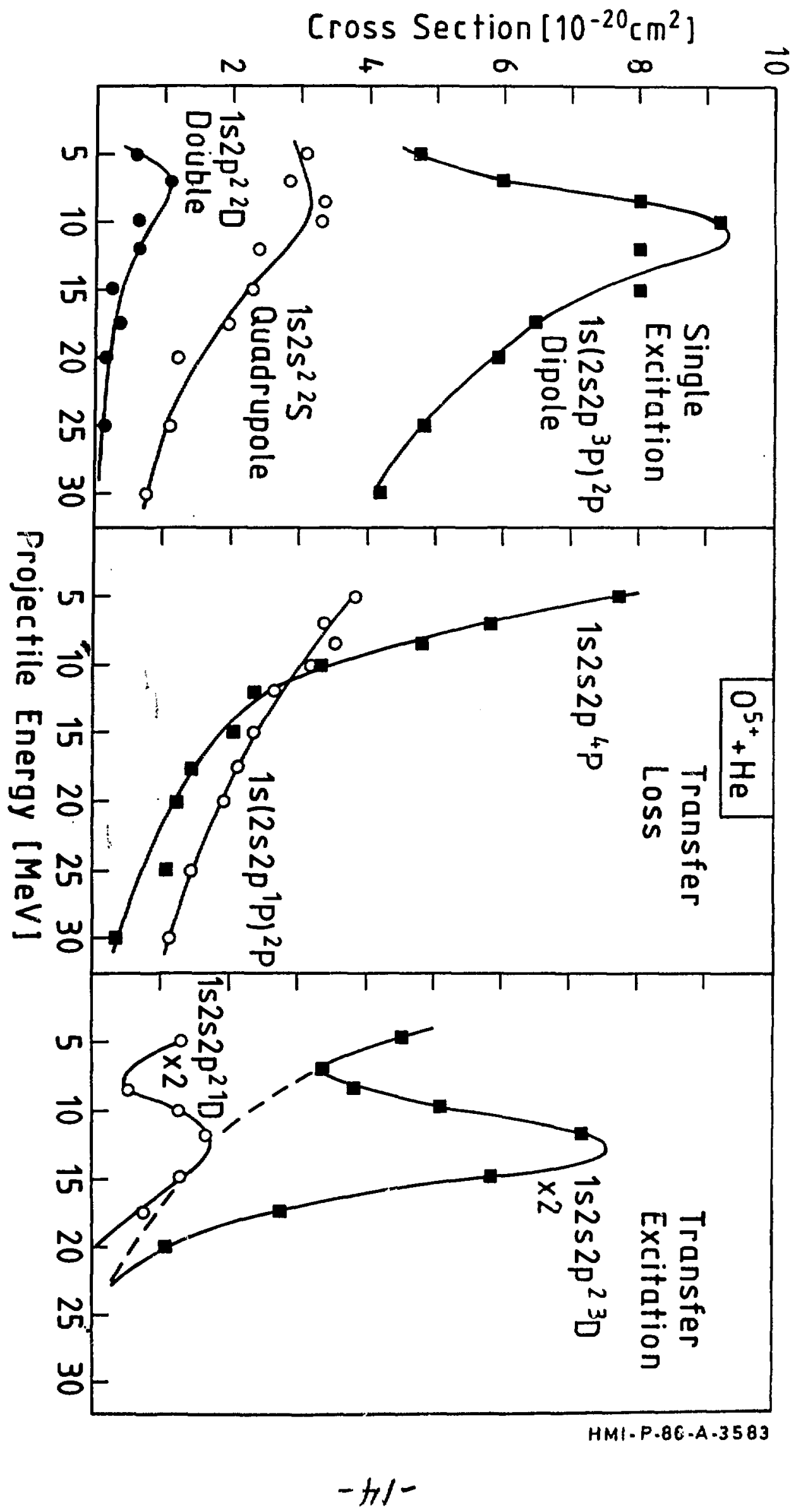




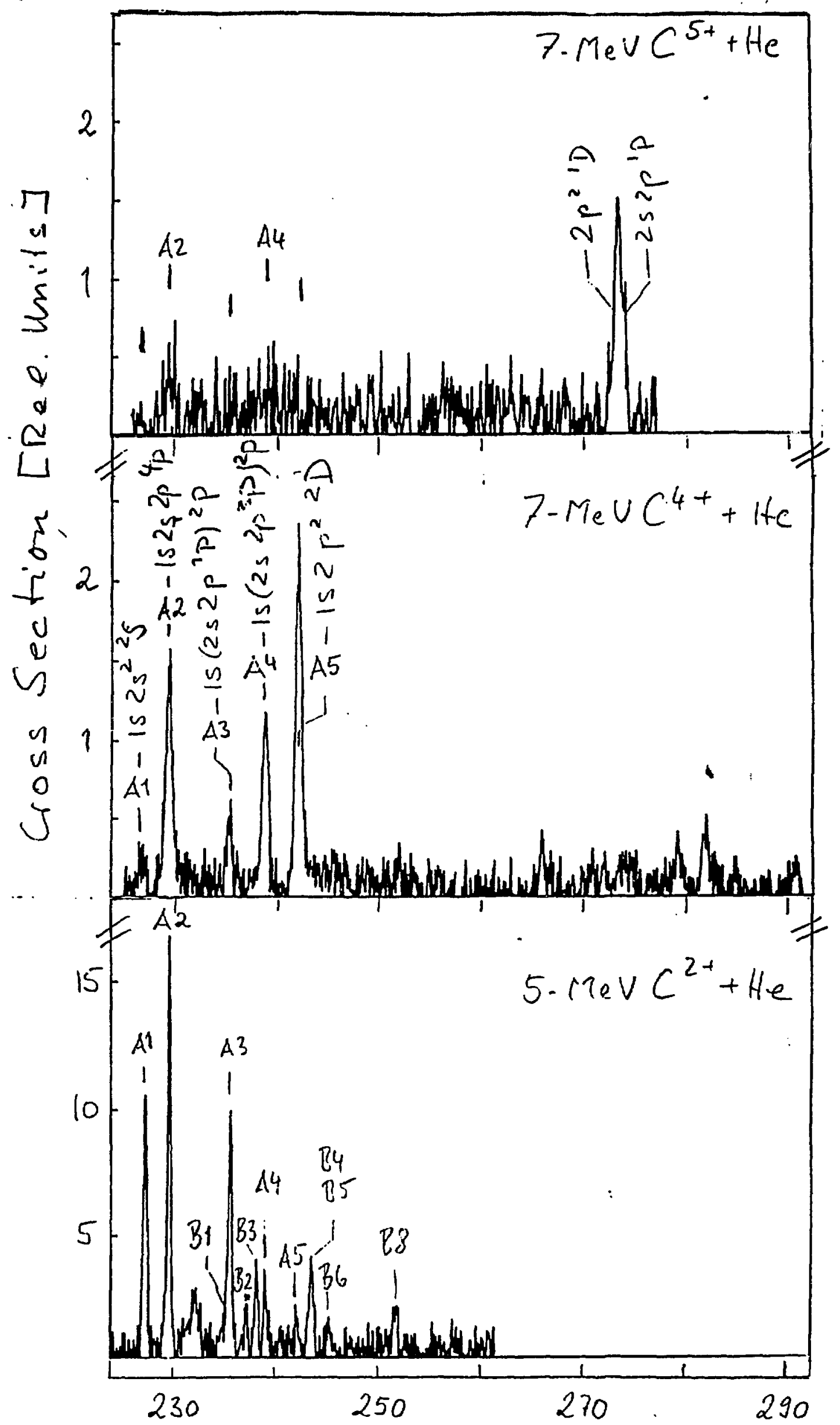

